\title{
Baseline geometry effects due to source structure in geodetic VLBI
}

\section{Julian Andres Mora-Diaz,; R. Heinkelmann, J. Anderson, T. Nilsson, and H. Schuh GFZ Helmholtz-Zentrum Potsdam}

E-mail: moraegfz-potsdam.de

\begin{abstract}
The technique of very long baseline interferometry (VLBI) uses knowledge about the positions of radio telescopes to measure celestial radio source positions with highest precision down to tens of microarcseconds for ground-based experiments. At the same time, VLBI measurements of stable, point-like celestial radio sources can be used to determine precise station positions down to the millimeter level. However, the majority of radio sources exhibit extended intrinsic structure at sub-milliarcsecond (or larger) scales that is time and frequency dependent and leads to systematic effects on the group delay observable. The classical approach to correct for structure if the radio sources are to be used for geodesy is to perform VLBI imaging, where the visibility function measured by the interferometer is the mapping of the specific intensity distribution of the source on the sky as a function of the interferometer baseline $(u, v)$ coordinates through a Fourier transform. Radio source images provide information about the sources' appearance and compactness. Ultimately, sources are classified according to their compactness, and for geodesy, the best sources are those of which core structure is compact and astrometrically stable: "defining sources". The sources exhibiting complex structure, "special handling sources", are always adjusted on the session level in the analysis, as they could otherwise corrupt the station position estimates. In this study we investigate the effects of source structure on the geodetic/astrometric results, i.e., radio source positions, by considering the different baseline-source geometries. For this purpose, we compare results obtained from astrometrically unstable sources observed with many long East-West baselines (to provide the highest angular resolution) with those from astrometrically stable sources.
\end{abstract}

12th European VLBI Network Symposium and Users Meeting

7-10 October 2014

Cagliari, Italy

\footnotetext{
*Speaker.

${ }^{\dagger}$ This research was funded by project DFG project ECORAS (HE 5937/2-1).
} 


\section{Introduction}

VLBI observations for precise astrometry and geodesy are successfully achieved whenever pointlike sources are observed. Most of the astronomical targets of radio telescopes show physical complex processes, which are not taken into account in the geodetic/astrometric analysis, and therefore the sources are not suitable objects for those purposes (Charlot 1990). As an example, within the second realization of the international celestial reference system (ICRF2) (Fey et al., 2009), sources were classified according to their significant position variations into three groups (Fey et al., 2009): defining sources: compact and astrometrically stable sources which are included in the axes definition and in the global No Net Rotation (NNR) condition; special handling sources: non-compact and most unstable sources, not estimated globally, and others: a mixture of stable and unstable sources; these are estimated globally but not considered in the axes definition. However, systematic effects of sources do not account for the physical evolution of radio galaxies and their complex phenomena e.g., emission of relativistic jets. Moreover, some authors have emphasized the importance of considering the structure of radio sources into the geodetic analysis (Cotton 1979, Thomas 1981, Charlot 1991, Campbell 1998), so that the geodetic community paid more attention to the source structure and the error this effect might be causing to the observables.

The correction to the group delay by source structure can be done if radio sources are long-term monitored, thus producing a good number of VLBI images, which are to be used for determining the structure index (Charlot 1990). Consequently, by knowing the structure index of the source it is conceivable to classify sources based upon their compactness. Currently there are some data bases ${ }^{1}$ that supply images of radio sources at the geodetic frequencies $(2.4 \mathrm{GHz}-8.3 \mathrm{GHz})$, though none of these archives contain enough images to estimate the evolution of the structure with time ${ }^{2}$.

\section{Classical correction and projection of baselines}

In a typical VLBI experiment, the visibility function measured by the interferometer is mapped from the intensity distribution of the source on the sky to a function of the interferometer which depends on the baseline $(u, v)$ by a Fourier transform. Geodetic VLBI experiments are not intended to produce radio source images; the main goal is to obtain accurate group delay observables to which different models and corrections are applied in order to study earth orientation parameters (EOPs), tectonic displacements, source positions, station positions, velocities, and so on.

In the present study, group delay observations at $8.4 \mathrm{GHz}$ of several radio sources $(0642+449$, OQ208, 4C39.25, and 1611-343) taken from typical geodetic experiments of $2003^{3}$ were reduced with the Vienna VLBI software (VieVS) (Böhm et al., 2012). Observations at $2.4 \mathrm{GHz}$ were simultaneously made with the aim of calibrating the $\mathrm{X}$ band data, hence having group delays free of ionospheric contributions (Böhm et al., 2012). Basically, VieVS software produces two solutions:

\footnotetext{
${ }^{1}$ To name some examples, the USNO VLBI S/X-band images database: http://www.usno.navy.mil/ USNO/astrometry/vlbi-products/rrfid/vlba_sx and the Bordeaux VLBI image database http:// vlbi.obs.u-bordeauxl.fr/

${ }^{2}$ It is worth noting that Petrov (2014) showed a very complete radio catalog, that can be found on http:// astrogeo.org/rfc/

${ }^{3}$ The choice of this year is not arbitrary. For the sources studied, there are many VLBI images found on the above databases and were useful for comparisons.
} 
the 'first' solution where clock breaks and large clock offsets are removed as well as quadratic terms of the clock, and the 'solution' where piecewise linear clock offsets are estimated and outliers are deleted. Because source structure effects can be very subtle, we assume the effects may be present in the first solution since the software is not capable of showing whether the source has structure or not. Later, a transformation from the terrestrial to the celestial reference system is done, allowing us to distinguish which baselines are aligned along jet axis (Fig. 1-right). Finally, we suppose that those baselines aligned along the jet are correlated with their high residuals of observation obtained from the first solution (in centimeters).
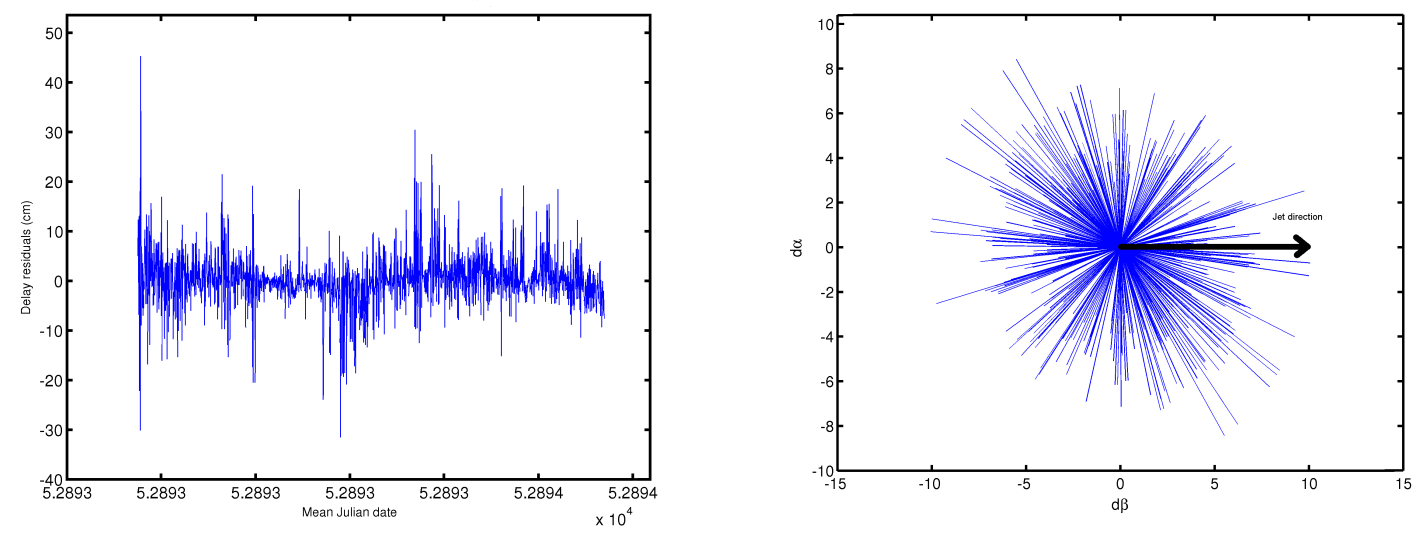

Figure 1: Left. Delay residuals (in centimeters) obtained in the first VieVS solution. Right. Baseline projection on the celestial reference frame (in arbitrary units).

\section{Results and conclusions}

Scatter plots of observation versus angle of baseline projected on the sky (position angle) were made for various sources with data binned (by averaging) within a window of 20 degrees (Fig. 2). Selected sources have different core-jet direction configurations that were examined from the USNO S/X band archive. We report results for sources 0642+449, OQ208, 4C39.25, and 1611343; the first is a compact source (i.e. no jet or second component detected) while the other have detected jets towards the west and south-west respectively, and the latter towards the south.

The plots show that some unstable sources have high residuals $(\gtrsim 1 \mathrm{~cm})$ and the stable source have lower values $(\lesssim 1 \mathrm{~cm})$. For sources OQ208 and 4C39.25, high residual values match with the position angle aligns to the jet direction (Fig. 2, top-right, bottom-left). Nevertheless, we expect 1611-343 to have high residual values for baselines along the south-west direction, thought there is no such correlation. Futhermore, there is not a clear pattern which allows us to determine the existence of a second component in unstable sources. Another interesting approach may be to analyse residuals as a function of baseline length, we will explore this idea in future work.

This study has provided an overview on how it is possible to handle source structure in the geodesy analysis by only having group delays. Further studies will model amplitudes, phases, and delays as a function of baseline length for each position angle and to model the source by considering its brightness distribution (Charlot 1990). 

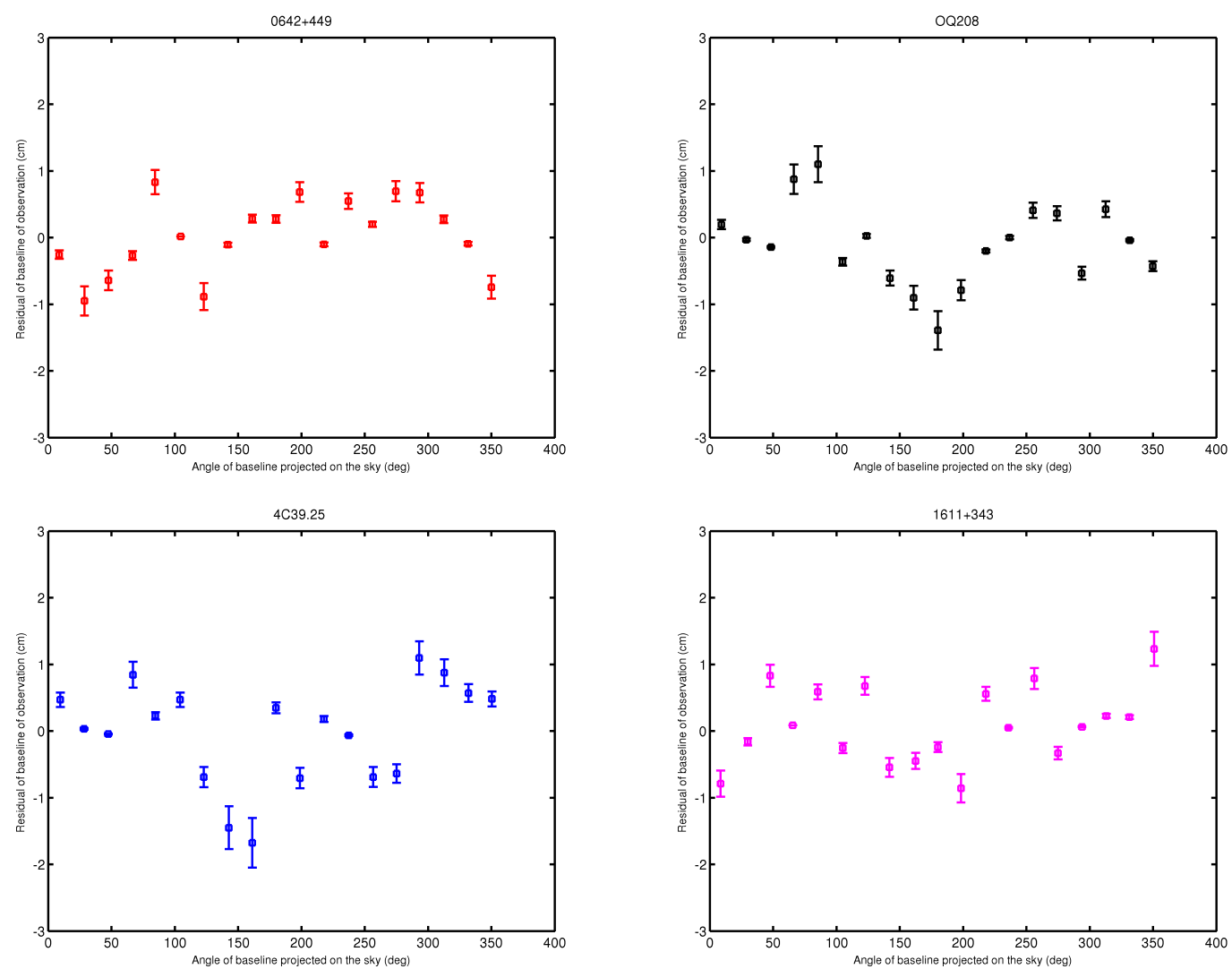

Figure 2: Binned data for sources: 0642+449 top-left (stable), OQ208 (unstable), 4C39.25 (unstable), and $1611+343$ (unstable). There is no clear pattern that allows to predict the existence of a second component.

\section{References}

[1] Charlot, P (1990). Radio-source structure in astrometric and geodetic very long baseline interferometry. Astronomical Journal, 99, No. 4.

[2] Fey A.L., Gordon D., Jacobs C.S. (eds.) (2009). The Second Realization of the International Celestial Reference Frame by Very Long Baseline Interferometry. IERS Technical Note No. 35. Presented on behalf of the IERS / IVS Working Group. Frankfurt am Main: Verlag des Bundesamts für Kartographie und Geodäsie, 204 pp., available at http://www.iers.org/TN35.

[3] Campbell, J., Schuh, H., and Zeppenfeld, G. (1998). The impact of VLBI on astrophysics and Geophysics, IAU Symposium No. 129, edited by M. J. Reid and J. M. Moran (Reidel, Drodrecht), p. 427.

[4] Cotton, W. E. (1979). In radio interferometry techniques for geodesy, NASA Conference No. 2115 (NASA Scientific and Technical Information Office. Springfield, Virginia), p 193.

[5] Petrov, L. (2014). Radio fundamental catalog (Poster), 12th European VLBI Network Symposium and users meeting, Cagliari, Italy, October 09-11.

[6] Böhm, J., Böhm, S., Nilsson, T. Pany, A., Plank, L., Spicakova, H., Teke, K., H Schuh (2011). The new Vienna VLBI Software VieVS, Springer Berlin Heidelberg, Geodesy for Planet Earth, pp 1007-1011.

[7] Thomas, J. B. (1980). NASA-JPL Publ. 80-84. 